\title{
InterCriteria Analyzis of Hybrid Ant Colony Optimization Algorithm for Multiple Knapsack Problem
}

\author{
Stefka Fidanova \\ Institute of Information and \\ Communication Technology \\ Bulgarian Academy of Sciences \\ Acad. G. Bonchev Str., bl. 25A, \\ Sofia, Bulgaria \\ E-mail: stefka@parallel.bas.bg
}

\author{
Maria Ganzha \\ System Research Institute \\ Polish Academy of Sciences \\ Warsaw, Poland \\ E-mail: maria.ganzha@ibspan.waw.pl
}

\author{
Olympia Roeva \\ Institute of Biophysics and \\ Biomedical Engineering \\ Bulgarian Academy of Sciences \\ Acad. G. Bonchev Str., bl. 105, \\ Sofia, Bulgaria \\ E-mail: olympia@biomed.bas.bg
}

\begin{abstract}
The local search procedure is a method for hybridization and improvement of the main algorithm, when complex problems are solved. It helps to avoid local optimums and to find faster the global one. In this paper we apply InterCriteria analysis (ICrA) on hybrid Ant Colony Optimization (ACO) algorithm for Multiple Knapsack Problem (MKP). The aim is to study the hybrid algorithm behavior comparing with traditional ACO algorithm. Based on the obtained numerical results and on the ICrA approach the efficiency and effectiveness of the proposed hybrid ACO, combined with appropriate local search procedure are confirmed.
\end{abstract}

Index Terms-Local Search, Ant Colony Optimization, InterCriteria analysis, Knapsack Problem

\section{INTRODUCTION}

$\mathbf{E}$ NGINEERING applications normally lead to complex decision make problems. Large scale problems can not be solved with traditional numerical methods. It is a challenge to develop a new techniques, which have simple structure and easy application, and can find near optimal solution even the information about the problem is incomplete. In most of the cases these problems are HP-hard.

Nature inspired methods are more appropriate for solving NP-hard optimization problem, than other methods, because they are flexible and use less computational resources. They are base on stochastic search. The most popular methods are Evolutionary algorithm [28], [45], which simulates the Darwinian evolutionary concept, Simulated Annealing [32] and Gravitation search algorithm [39], Tabu Search [42] and Interior Search [43]. The ideas for swarm-intelligence based algorithms come from behavior of animals in the natures. The representatives of this type of algorithms are Ant Colony Optimization [16], Bee Colony Optimization [29], Bat algorithm [46], Firefly algorithm [47], Particle Swarm Optimization [31], Gray Wolf algorithm [38] and so on.

Between the best methods for solving combinatorial optimization problems is Ant Colony Optimization (ACO). The impulse for this method comes from the behavior of real ants. They always find the shortest path from the food to the nest.
The ants leave a trail called a pheromone and follow the trail with the most concentrated pheromone.

The problem is represented with a help of a graph and the solutions are paths in a graph. The optimal solution for minimization problems is a shortest path, and for maximization problems is a longest path in a graph. The solution construction starts from random node of the graph and next nodes are included applying probabilistic rule. The pheromone is imitated by numerical information corresponding to the quality of the solution.

ACO is applied to many types of optimization problems. The idea for application of ant behavior for solving combinatorial optimization problems is done by Marco Dorigo twenty five years ago [15], [16], [18]. At the beginning it is applied on traveling salesman problem. Later it is successfully applied on a lot of complex optimization problems. During the years, various variants of ACO methodology was proposed: ant system [18]; elitist ants [18]; ant colony system [17]; max-min ant system [44]; rank-based ant system [18]; ant algorithm with additional reinforcement [21]. They differ in pheromone updating. For some of them is proven that they converge to the global optimum [18]. Fidanova et all [22]-[24] proposed semirandom start of the ants comparing several start strategies. The method can be adapted to dynamic changes of the problem in some complex biological problems [19], [20], [44].

Sometimes the metaheuristic algorithm, can not avoid local optimums. Appropriate Local Search (LS) procedure can help to escape them and to improve algorithm efficiency. We apply ACO on Multiple Knapsack Problem (MKP). A local search procedure, related with a specificity of MKP is constructed and combined with ACO to improve the algorithm performance and to avoid local optimums [24]. InterCriteria analysis (ICrA) is applied on the numerical results obtained by the traditional ACO and hybrid ACO in order to estimate the algorithms behavior. The approach ICrA has been applied for a large area of problems, e.g. [1], [2], [14], [25]. Published results show the applicability of the ICrA and the correctness of the approach. 
The rest of the paper is organized as follows: The definition of the MKP is in Section 2. ACO algorithm is presented in Section 3. Local Search procedure is described in Section 4. Short notes on ICrA approach are presented in Section 5. Numerical results and a discussion are in Section 6. Conclusion remarks are done in Section 7.

\section{Multiple Knapsack Problem}

In knapsack problem is given a set of items with fixed weights and values. The aim is to maximize the sum of the values of the items in the knapsack, while remaining within the capacity of the knapsack. Each item can be selected only ones.

Multiple Knapsack Problem (MKP) is a generalization of the single knapsack problem and instead to have only one knapsack, there are many knapsacks with diverse capacity. Each item is assigned to maximum one of the knapsacks without violating any of the knapsacks capacity. The purpose is to maximize the total profit of the items in the knapsacks.

MKP is a special case of the generalized assignment problem [36]. It is a representative of the subset problems. Economical, industrial and other types of problems can be represented by MKP. Resource allocation in distributed systems, capital budgeting, cargo loading and cutting stock problems [30] are some of the applications of the problem. One important real problem which is represented as MKP is patients scheduling [3]. MKP is related with bin packing problem where the size of the bins can be variable [40] and cutting stock problem for cut row materials [30]. Other application is multi-processor scheduling on uniformly related machines [35]. Other difficult problem which leads to MKP is crypto-systems and generating keys [30]. One early application of MKP is tests generation [27]. MKP is a model large set of binary problems with integer coefficients [33], [36].

MKP is NP-hard problem and normally is solved with some metaheuristic method such as genetic algorithm [37], tabue search [48], swarm intelligence [34], ACO algorithm [21], [26].

We will define MKP as resource allocation problem, where $m$ is the number of resources (the knapsacks) and $n$ is the number of the objects. The object $j$ has a profit $p_{j}$. Each resource has its own budget (knapsack capacity) and consumption $r_{i j}$ of resource $j$ by object $i$. The purpose is maximization of the profit within the limited budget.

The mathematical formulation of MKP can be as follows:

$$
\begin{gathered}
\max \sum_{j=1}^{n} p_{j} x_{j} \\
\text { subject to } \sum_{j=1}^{n} r_{i j} x_{j} \leq c_{i} \quad i=1, \ldots, m \\
x_{j} \in\{0,1\} \quad j=1, \ldots, n
\end{gathered}
$$

There are $m$ constraints in this problem, so MKP is also called $m$-dimensional knapsack problem. Let $I=\{1, \ldots, m\}$ and $J=\{1, \ldots, n\}$, with $c_{i} \geq 0$ for all $i \in I$. A well-stated MKP assumes that $p_{j}>0$ and $r_{i j} \leq c_{i} \leq \sum_{j=1}^{n} r_{i j}$ for all $i \in I$ and $j \in J$. Note that the $\left[r_{i j}\right]_{m \times n}$ matrix and $\left[c_{i}\right]_{m}$ vector are both non-negative.

The MKP partial solution is represented by $S=$ $\left\{i_{1}, i_{2}, \ldots, i_{j}\right\}$ and the last element included to $S, i_{j}$ is not used in the selection process for the next element. Thus the solution of MKP have not fixed length.

\section{Ant Colony Optimization Algorithm}

NP-hard problems require the use of huge resources and therefore cannot be solved by exact or traditional numerical methods, especially when they are large scale. We apply metaheuristic method aiming to find approximate solution using reasonable resources [18], [26].

Firs Marco Dorigo applies ideas coming from ants behavior to solve complicate optimization problems 30 years ago [16]. Some modifications are proposed by him and by other authors for algorithm improvement. The modifications concern pheromone updating [18]. The algorithm is problem dependent. Very important is representation of the problem by a graph. Thus the solutions represent paths in the graph. The ants look for an optimal path, taking in to account problem constraints.

The transition probability $P_{i, j}$, is a product of the heuristic information $\eta_{i, j}$ and the pheromone trail level $\tau_{i, j}$ related to the selection of node $j$ if the previous selected node is $i$, where $i, j=1, \ldots, n$.

$$
P_{i, j}=\frac{\tau_{i, j}^{a} \cdot \eta_{i, j}^{b}}{\sum_{k \in \text { Unused }} \tau_{i, k}^{a} \cdot \eta_{i, k}^{b}},
$$

where Unused is the set of unused nodes.

At the beginning the pheromone is initialized with a small constant value $\tau_{0}, 0<\tau_{0}<1$. Every time the ants build a solution, the pheromone is bring up to date [18].The elements of the graph with more pheromone are more tempting to the ants.

The main update rule for the pheromone is:

$$
\tau_{i, j} \leftarrow \rho \cdot \tau_{i, j}+\Delta \tau_{i, j},
$$

where parameter $\rho$ decreases the value of the pheromone, like evaporation in a nature decreases the quantity of old pheromone. $\Delta \tau_{i, j}$ is a new deposited pheromone, which depends on the value of the objective function, corresponding to this solution.

The first step, when ACO is applied on some combinatorial optimization problem is representation of the problem by graph. In our case the items are related with the nodes of the graph and the edges fully connect the nodes. The pheromone is deposited on the arcs of the graph.

Second step is construction of appropriate heuristic information. This step is very important, because the heuristic information is the main part of the transition probability function and the search process depends mainly on it. Normally the heuristic information is a combination of problem parameters. Let $s_{j}=\sum_{i=1}^{m} r_{i j}$. For heuristic information we use: 


$$
\eta_{i j}= \begin{cases}p_{j}^{d_{1}} / s_{j}^{d_{2}} & \text { if } s_{j} \neq 0 \\ p_{j}^{d_{1}} & \text { if } s_{j}=0\end{cases}
$$

where $d_{1}>0$ and $d_{2}>0$ are parameters. Hence the objects with greater profit and less average expenses will be more desirable. Thus is increased the probability to include more items and most profitable items. This can lead to maximization of the total profit, which is the objective of this problem.

\section{Local Search Procedure}

At times is used hybridization of the used method, for algorithm performance improvement. The goal is avoid some disadvantages of the main method. A possibility for hybridization is one of the methods to be basic and the other only helps to improve the solutions. Most used hybridization manner is local improvement or at the end of the iteration to apply some problem dependent local search procedure.

The Local Search (LS) procedure is used to perturbs current solution and to generate neighbor solutions [41]. LS generates neighbor solutions in a local set of neighbors. The best solution from the set is compared with the current solution. If it is better, it is accepted as a new current solution.

A LS procedure which is consistent with MKP has been developed and combined with ACO algorithm in our previous work [26]. The MKP solution is represented by binary string where 0 corresponds to not chosen item and 1 corresponds to item included in the solution. Two positions are randomly chosen. If the value of one of the positions is 0 we replace it with 1 and if the value of other position is 1 we replace it with 0 and vice versa. The feasibility of the new solution is verified. If the solution is feasible we compare it with the current (original) solution. The perturbed solution is accepted if its value of the objective function is greater, than of the original one.

We apply this LS procedure ones on each iteration on each solution, disregarding if the new constructed solution is better than current one or not. Thus the proposed LS works without significant increase of the used computational resources.

\section{INTERCRITERIA ANALYSIS}

Based on the apparatuses of index matrices [4], [6], [8], [9] and intuitionistic fuzzy sets (IFSs) [5], [7], [10], authors in [11] propose a new approach named InterCriteria analysis. Briefly presented, an intuitionistic fuzzy pair (IFP) [12] is an ordered pair of real non-negative numbers $\langle a, b\rangle$, where $a, b \in$ $[0,1]$ and $a+b \leq 1$, that is used as an evaluation of some object or process. According to [12], the components ( $a$ and b) of IFP might be interpreted as degrees of "membership" and "non-membership" to a given set, degrees of "agreement" and "disagreement", etc.
Let $O$ denotes the set of all objects being evaluated, and $C(O)$ is the set of values assigned by a given criteria $C$ (i.e., $C=C_{p}$ for some fixed $p$ ) to the objects, i.e.,

$$
\begin{aligned}
& O \stackrel{\text { def }}{=}\left\{O_{1}, O_{2}, O_{3}, \ldots, O_{n}\right\}, \\
& C(O) \stackrel{\text { def }}{=}\left\{C\left(O_{1}\right), C\left(O_{2}\right), C\left(O_{3}\right), \ldots, C\left(O_{n}\right)\right\} .
\end{aligned}
$$

Let $x_{i}=C\left(O_{i}\right)$. Then the following set can be defined:

$$
C^{*}(O) \stackrel{\text { def }}{=}\left\{\left\langle x_{i}, x_{j}\right\rangle \mid i \neq j \&\left\langle x_{i}, x_{j}\right\rangle \in C(O) \times C(O)\right\} .
$$

Further, if $x=C\left(O_{i}\right)$ and $y=C\left(O_{j}\right), x \prec y$ if $i<j$ will be written.

In order to find the agreement of different criteria, the vectors of all internal comparisons for each criterion are constructed, which elements fulfill one of the three relations $R, \bar{R}$ and $\widetilde{R}$. The nature of the relations is chosen such that for a fixed criterion $C$ and any ordered pair $\langle x, y\rangle \in C^{*}(O)$ :

$$
\begin{aligned}
& \langle x, y\rangle \in R \Leftrightarrow\langle y, x\rangle \in \bar{R}, \\
& \langle x, y\rangle \in \tilde{R} \Leftrightarrow\langle x, y\rangle \notin(R \cup \bar{R}), \\
& R \cup \bar{R} \cup \tilde{R}=C^{*}(O) .
\end{aligned}
$$

For example, if " $R$ " is the relation " $<$ ", then $\bar{R}$ is the relation ">", and vice versa.

When comparing two criteria the degree of "agreement"is determined as the number of matching components of the respective vectors (divided by the length of the vector for normalization purposes).

Let the respective degrees of "agreement" and "disagreement" are denoted by $\mu_{C, C^{\prime}}$ and $\nu_{C, C^{\prime}}$. In the most of the obtained pairs $\left\langle\mu_{C, C^{\prime}}, \nu_{C, C^{\prime}}\right\rangle$, the sum $\mu_{C, C^{\prime}}+\nu_{C, C^{\prime}}$ is equal to 1 . However, there may be some pairs, for which this sum is less than 1 . The difference

$$
\pi_{C, C^{\prime}}=1-\mu_{C, C^{\prime}}-\nu_{C, C^{\prime}}
$$

is considered as a degree of "uncertainty.

\section{Vi. Computational Results and Discussion}

The proposed hybrid ACO algorithm for MKP is tested on 10 test MKP instances from Operational Research Library "OR-Library" available within WWW access at http://people.brunel.ac.uk/ mastjjb/jeb/info.html. Every test problem consists of 100 items and 10 constraints/knapsacks. We prepare a software, which realizes our hybrid algorithm. The software is coded in $C++$ program language and is run on Pentium desktop computer at $2.8 \mathrm{GHz}$ with $4 \mathrm{~GB}$ of memory. The ACO algorithm parameters are fixed experimentally as follows:

- Number of iterations $=300$, Number of ants $=20$;

- $\rho=0.5, \tau_{0}=0.5$;

- $a=1, b=1$ and $d_{1}=1$.

We perform 30 independent runs with every one of the test instances, because the algorithm is stochastic and to guarantee the robustness of the average results. We apply ANOVA test for statistical analysis and thus we guarantee the significance of the difference between the average results. The names of 
TABLE I: Test instances

\begin{tabular}{|c|c|c|}
\hline \multirow{2}{*}{ Instance } & \multicolumn{2}{|c|}{ Name } \\
\cline { 2 - 3 } & Hybrid ACO & Traditional ACO \\
\hline MKP $100 \times 10-01$ & $P 1_{h}$ & $P 1_{t}$ \\
\hline MKP $100 \times 10-02$ & $P 2_{h}$ & $P 2_{t}$ \\
\hline MKP $100 \times 10-03$ & $P 3_{h}$ & $P 3_{t}$ \\
\hline MKP $100 \times 10-04$ & $P 4_{h}$ & $P 4_{t}$ \\
\hline MKP $100 \times 10-05$ & $P 5_{h}$ & $P 5_{t}$ \\
\hline MKP $100 \times 10-06$ & $P 6_{h}$ & $P 6_{t}$ \\
\hline MKP $100 \times 10-07$ & $P 7_{h}$ & $P 7_{t}$ \\
\hline MKP $100 \times 10-08$ & $P 8_{h}$ & $P 8_{t}$ \\
\hline MKP $100 \times 10-09$ & $P 9_{h}$ & $P 9_{t}$ \\
\hline MKP $100 \times 10-10$ & $P 10_{h}$ & $P 10_{t}$ \\
\hline
\end{tabular}

TABLE II: Traditional ACO performance

\begin{tabular}{|c|c|c|c|c|c|c|c|c|c|c|}
\hline & $P 1_{t}$ & $P 2_{t}$ & $P 3_{t}$ & $P 4_{t}$ & $P 5_{t}$ & $P 6_{t}$ & $P 7_{t}$ & $P 8_{t}$ & $P 9_{t}$ & $P 10_{t}$ \\
\hline run1 & 22089 & 22452 & 20936 & 21481 & 21751 & 21810 & 21537 & 21634 & 22213 & 40594 \\
\hline run2 & 21954 & 22055 & 20966 & 21318 & 21606 & 21864 & 21659 & 21596 & 22398 & 40701 \\
\hline run3 & 21935 & 21912 & 21023 & 21318 & 21463 & 21912 & 21526 & 21516 & 22065 & 40647 \\
\hline run4 & 22030 & 21914 & 20732 & 21556 & 21519 & 21903 & 21470 & 21337 & 22191 & 40617 \\
\hline run5 & 21875 & 21990 & 21120 & 21451 & 21903 & 21970 & 21360 & 21689 & 22152 & 40489 \\
\hline run6 & 21970 & 21999 & 21114 & 21619 & 21736 & 21756 & 21426 & 21729 & 22125 & 40646 \\
\hline run7 & 21974 & 21990 & 21085 & 21740 & 21641 & 21654 & 21522 & 21515 & 22398 & 40714 \\
\hline run8 & 22041 & 22120 & 21032 & 21918 & 21811 & 22053 & 21584 & 21550 & 22109 & 40550 \\
\hline run9 & 21893 & 21924 & 21187 & 21335 & 21716 & 21864 & 21587 & 21725 & 22398 & 40581 \\
\hline run10 & 21984 & 22104 & 20822 & 21719 & 21767 & 21864 & 21509 & 21550 & 22078 & 40594 \\
\hline run11 & 21787 & 21950 & 21042 & 21661 & 21673 & 22047 & 21595 & 22067 & 22398 & 40659 \\
\hline run12 & 21916 & 22675 & 21182 & 21629 & 21818 & 21834 & 21426 & 21550 & 22101 & 40646 \\
\hline run13 & 22031 & 22027 & 20869 & 21490 & 21843 & 22123 & 21466 & 21508 & 22398 & 40584 \\
\hline run14 & 22188 & 21975 & 21203 & 21736 & 21811 & 21864 & 21601 & 21550 & 22398 & 40627 \\
\hline run15 & 21889 & 22119 & 21204 & 21740 & 21716 & 21824 & 21509 & 21573 & 22086 & 40515 \\
\hline run16 & 22009 & 22101 & 20877 & 21705 & 21952 & 21779 & 21409 & 21506 & 22039 & 40498 \\
\hline run17 & 21880 & 21990 & 21085 & 21531 & 21736 & 21713 & 21394 & 21579 & 22398 & 40680 \\
\hline run18 & 21958 & 22102 & 20872 & 21335 & 21811 & 22053 & 21596 & 21550 & 22105 & 40589 \\
\hline run19 & 22015 & 21963 & 20841 & 21861 & 21581 & 21864 & 21624 & 21729 & 22324 & 40404 \\
\hline run20 & 22054 & 22027 & 21007 & 21815 & 21679 & 22140 & 21392 & 21729 & 22398 & 40367 \\
\hline run21 & 22093 & 22027 & 20833 & 21607 & 21811 & 21816 & 21509 & 21496 & 22039 & 40496 \\
\hline run22 & 22074 & 22065 & 20960 & 21701 & 21711 & 21903 & 21509 & 21496 & 22398 & 40737 \\
\hline run23 & 22003 & 22027 & 20976 & 21759 & 21685 & 21864 & 21522 & 21339 & 22039 & 40594 \\
\hline run24 & 22169 & 22005 & 21003 & 21490 & 21735 & 21898 & 21511 & 21520 & 22156 & 40317 \\
\hline run25 & 22091 & 22106 & 20808 & 21964 & 21622 & 21840 & 21434 & 21614 & 22398 & 40664 \\
\hline run26 & 21945 & 21899 & 21103 & 21335 & 21417 & 22053 & 21426 & 21516 & 22059 & 40319 \\
\hline run27 & 22086 & 22196 & 21069 & 21774 & 21944 & 22241 & 21590 & 21629 & 22398 & 40728 \\
\hline run28 & 21926 & 21975 & 21003 & 21437 & 21922 & 21864 & 21531 & 21503 & 22398 & 40636 \\
\hline run29 & 21929 & 22177 & 20799 & 21681 & 21736 & 21907 & 21479 & 21530 & 22398 & 40498 \\
\hline run30 & 22030 & 21931 & 20925 & 22061 & 21434 & 21864 & 21509 & 21366 & 22398 & 40756 \\
\hline
\end{tabular}

the test instances are presented in Table I. In Table II and Table III observed numerical results for all 30 runs are listed.

In Table IV are reported average results for every one of the test instances over 30 runs. We compare ACO algorithm combined with local search procedure (hybrid ACO) with traditional ACO algorithm. On the last row is reported average computational time, in seconds, of the two variants of ACO algorithm.

Table IV shows that for eight of ten instances hybrid ACO algorithm outperforms the traditional one. For the instances MKP $100 \times 10-02$ and MKP $100 \times 10-10$ the results are statistically the same. The main problem with hybrid algo- 
TABLE III: Hybrid ACO performance

\begin{tabular}{|c|c|c|c|c|c|c|c|c|c|c|}
\hline & $P 1_{h}$ & $P 2_{h}$ & $P 3_{h}$ & $P 4_{h}$ & $P 5_{h}$ & $P 6_{h}$ & $P 7_{h}$ & $P 8_{h}$ & $P 9_{h}$ & $P 10_{h}$ \\
\hline run1 & 22206 & 22047 & 21292 & 21885 & 21811 & 21940 & 21509 & 22004 & 22270 & 40647 \\
\hline run2 & 21968 & 22186 & 21089 & 21962 & 21811 & 21957 & 21509 & 21616 & 22097 & 40557 \\
\hline run3 & 21970 & 22074 & 21233 & 22139 & 21716 & 21934 & 21530 & 21550 & 22087 & 40598 \\
\hline run4 & 22130 & 22028 & 21687 & 21701 & 21811 & 22024 & 21415 & 21729 & 22294 & 40679 \\
\hline run5 & 22107 & 22168 & 21020 & 21885 & 21811 & 22047 & 21522 & 21729 & 22285 & 40647 \\
\hline run6 & 22138 & 22027 & 21222 & 21736 & 21798 & 21980 & 21522 & 21551 & 22257 & 40522 \\
\hline run7 & 22367 & 22027 & 21416 & 21962 & 21811 & 21900 & 21437 & 21729 & 22140 & 40538 \\
\hline run8 & 22051 & 22028 & 21261 & 21420 & 21790 & 22048 & 21509 & 21648 & 22125 & 40710 \\
\hline run9 & 21867 & 22104 & 20861 & 21780 & 21811 & 21985 & 21531 & 21729 & 22398 & 40710 \\
\hline run 10 & 21910 & 22027 & 21090 & 21666 & 21811 & 21987 & 21537 & 21729 & 22398 & 40647 \\
\hline run11 & 22133 & 22027 & 20758 & 21854 & 21844 & 22011 & 21655 & 21729 & 22398 & 40583 \\
\hline run12 & 22164 & 22074 & 20848 & 21885 & 21798 & 22053 & 21330 & 21653 & 22154 & 40662 \\
\hline run13 & 21949 & 22044 & 21090 & 22099 & 21736 & 21987 & 21409 & 21729 & 22398 & 40664 \\
\hline run14 & 22067 & 22102 & 20954 & 21921 & 21798 & 21987 & 21587 & 21729 & 22117 & 40683 \\
\hline run15 & 21889 & 22027 & 21236 & 21801 & 21811 & 22113 & 21509 & 21729 & 22069 & 40689 \\
\hline run16 & 21999 & 22213 & 21185 & 21561 & 21811 & 21900 & 21509 & 21650 & 22429 & 40636 \\
\hline run17 & 21926 & 22065 & 21017 & 22174 & 21914 & 21937 & 21509 & 21550 & 22191 & 40489 \\
\hline run18 & 21914 & 22027 & 21097 & 21542 & 21855 & 21864 & 21509 & 21550 & 22247 & 40714 \\
\hline run19 & 22008 & 22028 & 20953 & 21490 & 21796 & 22053 & 21624 & 21683 & 22398 & 40677 \\
\hline run20 & 21840 & 22151 & 21166 & 21893 & 21804 & 21987 & 21418 & 21729 & 22193 & 40728 \\
\hline run21 & 21993 & 22155 & 20839 & 21656 & 21811 & 21891 & 21584 & 21550 & 22398 & 40565 \\
\hline run22 & 22130 & 22106 & 21134 & 21962 & 21798 & 22063 & 21533 & 21658 & 22479 & 40742 \\
\hline run23 & 21958 & 22060 & 20881 & 21885 & 21811 & 21987 & 21426 & 21729 & 22152 & 40503 \\
\hline run24 & 22014 & 22027 & 21373 & 22023 & 21811 & 21924 & 21511 & 21729 & 22152 & 40514 \\
\hline run25 & 22072 & 22027 & 20925 & 21953 & 21811 & 21987 & 21509 & 21697 & 22123 & 40650 \\
\hline run26 & 22088 & 22104 & 20857 & 22052 & 21974 & 21987 & 21509 & 21550 & 22429 & 40751 \\
\hline run27 & 21928 & 22027 & 20934 & 21864 & 21811 & 21987 & 21509 & 21550 & 22218 & 40658 \\
\hline run28 & 21933 & 22110 & 20916 & 21885 & 21811 & 22121 & 21509 & 21689 & 22398 & 40499 \\
\hline run29 & 21970 & 22027 & 21281 & 21793 & 21832 & 21987 & 21509 & 21729 & 22398 & 40598 \\
\hline run30 & 21993 & 22027 & 21064 & 21951 & 21811 & 22050 & 21509 & 21550 & 22479 & 40540 \\
\hline
\end{tabular}

TABLE IV: Comparison of ACO performance

\begin{tabular}{|l|c|c|}
\hline Instance & Hybrid ACO & Traditional ACO \\
\hline MKP $100 \times 10-01$ & $\mathbf{2 2 0 2 2 . 7 3}$ & 21989.43 \\
\hline MKP $100 \times 10-02$ & 22071.46 & 22081.36 \\
\hline MKP $100 \times 10-03$ & $\mathbf{2 1 0 8 9 . 3}$ & 21027.63 \\
\hline MKP $100 \times 10-04$ & $\mathbf{2 1 8 4 6}$ & 21635.3 \\
\hline MKP $100 \times 10-05$ & $\mathbf{2 1 8 1 4 . 3}$ & 21717.3 \\
\hline MKP $100 \times 10-06$ & $\mathbf{2 1 9 8 9 . 2 6}$ & 21869.73 \\
\hline MKP $100 \times 10-07$ & $\mathbf{2 1 5 0 6 . 2 6}$ & 21477.3 \\
\hline MKP $100 \times 10-08$ & $\mathbf{2 1 6 7 2 . 5 3}$ & 21606.43 \\
\hline MKP $100 \times 10-09$ & $\mathbf{2 2 2 7 2 . 3 6}$ & 22257 \\
\hline MKP $100 \times 10-10$ & 40626.66 & 40623.26 \\
\hline computational time & $64.052 \mathrm{~s}$ & $65.552 \mathrm{~s}$ \\
\hline
\end{tabular}

rithms, when some global method is combined with local search procedure, is increasing of computational time. We try to propose efficient and in a same time less time consuming local search. We only change randomly chosen position in a solution to 0 , if it is 1 and another randomly chosen position to
1 if it is 0 . Thus is generated only one neighbor solution. If this solution is better than the current one, it is accepted and used for pheromone updating instead of the solution constructed by the ant. We apply this procedure to each of the solutions. As is seen from Table IV the increase of computational time, when our local search is applied is only $2.34 \%$.

Thus we can conclude that proposed local search procedure is efficient and effective. The algorithm performance is improved, without significant increase of the computational time.

To support these claims, the obtained numerical results were analyzed using ICrA. The input matrix for ICrA has the following form index matrix Table $\mathrm{V}$ :

The obtained by ICrA results are listed in Table VI ( $\mu$ values) and Table VII ( $\nu$-values). The $\pi$-values are also presented (see Table VIII). The results between the same instances but for different ACO algorithms are presented. For example, relations between $P 1_{t}-P 1_{h}, P 2_{t}-P 2_{h}, P 3_{t}-P 3_{h}$, etc. are considered for further analysis (presented in bold results in Tables VI, VII and VIII).

According to [13] the results show that the considered 
TABLE V: Index matrix for ICrA

\begin{tabular}{l|cccc} 
& run1 & run2 & $\ldots$ & run30 \\
\hline$P 1_{t}$ & $\operatorname{val}_{P 1_{t, 1}}$ & $\operatorname{val}_{P 1_{t, 2}}$ & $\vdots$ & $\operatorname{val}_{P 1_{t, 30}}$ \\
$P 2_{t}$ & $\operatorname{val}_{P 2_{t, 1}}$ & $\operatorname{val}_{P 2_{t, 2}}$ & $\vdots$ & $\operatorname{val}_{P 2_{t, 30}}$ \\
$\vdots$ & $\vdots$ & $\vdots$ & $\ddots$ & $\vdots$ \\
$P 10_{t}$ & $\operatorname{val}_{P 10_{t, 1}}$ & $\operatorname{val}_{P 10_{t, 2}}$ & $\vdots$ & $\operatorname{val}_{P 10_{t, 30}}$ \\
$P 1_{h}$ & $\operatorname{val}_{P 1_{h, 1}}$ & $\operatorname{val}_{P 1_{h, 2}}$ & $\vdots$ & $\operatorname{val}_{P 1_{h, 30}}$ \\
$P 2_{h}$ & $\operatorname{val}_{P 2_{h, 1}}$ & $\operatorname{val}_{P 2_{h, 2}}$ & $\vdots$ & $\operatorname{val}_{P 2_{h, 30}}$ \\
$\vdots$ & $\vdots$ & $\vdots$ & $\ddots$ & $\vdots$ \\
$P 10_{h}$ & $\operatorname{val}_{P 10_{h, 1}}$ & $\operatorname{val}_{P 10_{h, 2}}$ & $\vdots$ & $\operatorname{val}_{P 10_{h, 30}}$
\end{tabular}

TABLE VI: Degree of agreement $-\mu_{C, C^{\prime}}$-values

\begin{tabular}{|l|c|c|c|c|c|c|c|c|c|c|}
\hline$\mu$ & $P 1_{t}$ & $P 2_{t}$ & $P 3_{t}$ & $P 4_{t}$ & $P 5_{t}$ & $P 6_{t}$ & $P 7_{t}$ & $P 8_{t}$ & $P 9_{t}$ & $P 10_{t}$ \\
\hline$P 1_{h}$ & $\mathbf{0 . 5 5}$ & 0.46 & 0.51 & 0.5 & 0.45 & 0.38 & 0.45 & 0.46 & 0.37 & 0.55 \\
\hline$P 2_{h}$ & 0.42 & $\mathbf{0 . 3 7}$ & 0.48 & 0.3 & 0.48 & 0.39 & 0.36 & 0.37 & 0.34 & 0.36 \\
\hline$P 3_{h}$ & 0.57 & 0.57 & $\mathbf{0 . 4 4}$ & 0.5 & 0.46 & 0.46 & 0.46 & 0.43 & 0.39 & 0.47 \\
\hline$P 4_{h}$ & 0.47 & 0.39 & 0.55 & $\mathbf{0 . 4}$ & 0.35 & 0.47 & 0.4 & 0.41 & 0.51 & 0.61 \\
\hline$P 5_{h}$ & 0.25 & 0.34 & 0.33 & 0.27 & $\mathbf{0 . 3 2}$ & 0.36 & 0.35 & 0.34 & 0.34 & 0.34 \\
\hline$P 6_{h}$ & 0.4 & 0.45 & 0.5 & 0.57 & 0.43 & $\mathbf{0 . 5}$ & 0.44 & 0.46 & 0.46 & 0.48 \\
\hline$P 7_{h}$ & 0.41 & 0.3 & 0.44 & 0.38 & 0.4 & 0.38 & $\mathbf{0 . 5 2}$ & 0.51 & 0.36 & 0.38 \\
\hline$P 8_{h}$ & 0.4 & 0.42 & 0.38 & 0.42 & 0.4 & 0.37 & 0.39 & $\mathbf{0 . 4 2}$ & 0.42 & 0.29 \\
\hline$P 9_{h}$ & 0.45 & 0.38 & 0.35 & 0.44 & 0.49 & 0.49 & 0.39 & 0.39 & $\mathbf{0 . 4 2}$ & 0.41 \\
\hline$P 10_{h}$ & 0.55 & 0.52 & 0.5 & 0.52 & 0.46 & 0.63 & 0.48 & 0.54 & 0.38 & $\mathbf{0 . 3 8}$ \\
\hline
\end{tabular}

TABLE VII: Degree of disagreement $-\nu_{C, C^{\prime} \text {-values }}$

\begin{tabular}{|l|c|c|c|c|c|c|c|c|c|c|}
\hline$\nu$ & $P 1_{t}$ & $P 2_{t}$ & $P 3_{t}$ & $P 4_{t}$ & $P 5_{t}$ & $P 6_{t}$ & $P 7_{t}$ & $P 8_{t}$ & $P 9_{t}$ & $P 10_{t}$ \\
\hline$P 1_{h}$ & $\mathbf{0 . 4 4}$ & 0.51 & 0.47 & 0.48 & 0.52 & 0.54 & 0.52 & 0.5 & 0.4 & 0.43 \\
\hline$P 2_{h}$ & 0.44 & $\mathbf{0 . 4 6}$ & 0.38 & 0.56 & 0.37 & 0.4 & 0.48 & 0.46 & 0.38 & 0.49 \\
\hline$P 3_{h}$ & 0.43 & 0.4 & $\mathbf{0 . 5 5}$ & 0.49 & 0.52 & 0.47 & 0.5 & 0.54 & 0.4 & 0.51 \\
\hline$P 4_{h}$ & 0.49 & 0.55 & 0.42 & $\mathbf{0 . 5 5}$ & 0.59 & 0.43 & 0.54 & 0.53 & 0.26 & 0.36 \\
\hline$P 5_{h}$ & 0.46 & 0.36 & 0.38 & 0.43 & $\mathbf{0 . 3 7}$ & 0.35 & 0.37 & 0.34 & 0.27 & 0.37 \\
\hline$P 6_{h}$ & 0.51 & 0.45 & 0.41 & 0.33 & 0.46 & $\mathbf{0 . 3 6}$ & 0.44 & 0.42 & 0.31 & 0.43 \\
\hline$P 7_{h}$ & 0.41 & 0.5 & 0.37 & 0.43 & 0.41 & 0.4 & $\mathbf{0 . 2 7}$ & 0.28 & 0.34 & 0.43 \\
\hline$P 8_{h}$ & 0.37 & 0.35 & 0.39 & 0.35 & 0.36 & 0.36 & 0.37 & $\mathbf{0 . 3 3}$ & 0.24 & 0.47 \\
\hline$P 9_{h}$ & 0.47 & 0.53 & 0.57 & 0.48 & 0.41 & 0.4 & 0.52 & 0.51 & $\mathbf{0 . 3 4}$ & 0.51 \\
\hline$P 0_{h}$ & 0.43 & 0.44 & 0.48 & 0.45 & 0.5 & 0.29 & 0.47 & 0.42 & 0.39 & $\mathbf{0 . 6}$ \\
\hline
\end{tabular}

criteria pairs, are in dissonance or in strong dissonance. This means that the both compared ACO algorithms (hybrid and traditional ones) performed differently in case of all 10 various instances.

The results obtained form ICrA are correct and reliable, taking into account the observed values of $\pi_{C, C^{\prime}}$-values. Only for relations between $P 5_{t}-P 5_{h}, P 8_{t}-P 8_{h}$ and $P 9_{t}-P 9_{h}$,

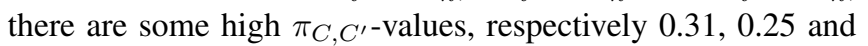
0.24 . The obtained estimates for the degree of agreement and the degree of disagreement have a high degree of uncertainty.

\section{CONCLUSION}

In this paper we propose hybrid ACO algorithm for solving MKP. The algorithm is combination of traditional ACO algorithm and local search procedure. Proposed algorithm is tested on 10 benchmark MKP. The achieved results show the efficiency and effectiveness of the proposed local search procedure. The hybrid algorithm performs better than the traditional one, while the calculation time increases only with $2.34 \%$.

Obtained results are analyzed by ICrA approach. The anal- 
TABLE VIII: Degree of uncertainty $-\pi_{C, C^{\prime} \text {-values }}$

\begin{tabular}{|l|c|c|c|c|c|c|c|c|c|c|}
\hline$\pi$ & $P 1_{t}$ & $P 2_{t}$ & $P 3_{t}$ & $P 4_{t}$ & $P 5_{t}$ & $P 6_{t}$ & $P 7_{t}$ & $P 8_{t}$ & $P 9_{t}$ & $P 10_{t}$ \\
\hline$P 1_{h}$ & $\mathbf{0 . 0 1}$ & 0.03 & 0.02 & 0.02 & 0.03 & 0.08 & 0.03 & 0.04 & 0.23 & 0.02 \\
\hline$P 2_{h}$ & 0.14 & $\mathbf{0 . 1 7}$ & 0.14 & 0.14 & 0.15 & 0.21 & 0.16 & 0.17 & 0.28 & 0.15 \\
\hline$P 3_{h}$ & 0 & 0.03 & $\mathbf{0 . 0 1}$ & 0.01 & 0.02 & 0.07 & 0.04 & 0.03 & 0.21 & 0.02 \\
\hline$P 4_{h}$ & 0.04 & 0.06 & 0.03 & $\mathbf{0 . 0 5}$ & 0.06 & 0.1 & 0.06 & 0.06 & 0.23 & 0.03 \\
\hline$P 5_{h}$ & 0.29 & 0.3 & 0.29 & 0.3 & $\mathbf{0 . 3 1}$ & 0.29 & 0.28 & 0.32 & 0.39 & 0.29 \\
\hline$P 6_{h}$ & 0.09 & 0.1 & 0.09 & 0.1 & 0.11 & $\mathbf{0 . 1 4}$ & 0.12 & 0.12 & 0.23 & 0.09 \\
\hline$P 7_{h}$ & 0.18 & 0.2 & 0.19 & 0.19 & 0.19 & 0.22 & $\mathbf{0 . 2 1}$ & 0.21 & 0.3 & 0.19 \\
\hline$P 8_{h}$ & 0.23 & 0.23 & 0.23 & 0.23 & 0.24 & 0.27 & 0.24 & $\mathbf{0 . 2 5}$ & 0.34 & 0.24 \\
\hline$P 9_{h}$ & 0.08 & 0.09 & 0.08 & 0.08 & 0.1 & 0.11 & 0.09 & 0.1 & $\mathbf{0 . 2 4}$ & 0.08 \\
\hline$P 10_{h}$ & 0.02 & 0.04 & 0.02 & 0.03 & 0.04 & 0.08 & 0.05 & 0.04 & 0.23 & $\mathbf{0 . 0 2}$ \\
\hline
\end{tabular}

ysis shows that the both algorithms performs differently for the considered 10 instances, i.e. the behavior of the proposed hybrid ACO is importantly different from that of the traditional ACO algorithm, or the local search procedure perturbs significantly the search process.

Through the application of ICrA approach the efficiency and effectiveness of the proposed hybrid ACO agorithm are confirmed.

\section{ACKNOWLEDGMENT}

The development of the proposed hybrid ACO algorithm with local search procedure has been funded by the Grant DFNI DN 12/5 and Grant No BG05M2OP001-1.001-0003, financed by the Science and Education for Smart Growth Operational Program and co-financed by the European Union through the European structural and Investment funds. The study of ACO algorithms behavior based on ICrA approach has been funded by the Grant KP-06-N22/1.

Author Contributions: The authors contributed equally to the work.

\section{REFERENCES}

[1] M. Angelova, O. Roeva and T. Pencheva. InterCriteria Analysis of Crossover and Mutation Rates Relations in Simple Genetic Algorithm. In Proceedings of the 2015 Federated Conference on Computer Science and Information Systems, Vol. 5, pages 419-424, 2015.

[2] Antonov A., Dependencies Between Model Indicators of the Basic and the Specialized Speed in Hockey Players Aged 13-14, Trakia Journal of Sciences, Vol. 18, Suppl. 1, pp 647-657, 2020.

[3] Idil Arsik, Pinar Keskinocak, Jennifer Coppola, Kirthana Hampapur, Yitong He, Haozheng Jiang,Dani Regala, Nick Tailhardat, and Kristin Goin. Effective and equitable appointment scheduling in rehabilitation centers.INFORMS Annual Meeting 2017, Oct. 22-25 2017.

[4] K. Atanassov. Index matrices: Towards an augmented matrix calculus. Springer International Publishing Switzerland, 2014.

[5] K. Atanassov. Intuitionistic Fuzzy Sets. VII ITKR Session, Sofia, 20-23 June 1983, Reprinted: International Journal Bioautomation, 20(S1):S1S6, 2016.

[6] K. Atanassov. Generalized index matrices. Comptes rendus de l'Academie bulgare des Sciences, 40(11):15-18, 1987.

[7] K. Atanassov. On intuitionistic fuzzy sets theory. Springer, Berlin, 2012.

[8] K. Atanassov. On index matrices, Part 1: Standard cases. Advanced Studies in Contemporary Mathematics, 20(2):291-302, 2010).

[9] K. Atanassov. On index matrices, Part 2: Intuitionistic fuzzy case. Proceedings of the Jangjeon Mathematical Society, 13(2):121-126, 2010.
[10] K. Atanassov. Review and New Results on Intuitionistic Fuzzy Sets, Mathematical Foundations of Artificial Intelligence Seminar, Sofia, 1988, Preprint IM-MFAIS-1-88. Reprinted: International Journal Bioautomation, 20(S1):S7-S16, 2016.

[11] K. Atanassov, D. Mavrov and V. Atanassova. Intercriteria decision making: A new approach for multicriteria decision making, based on index matrices and intuitionistic fuzzy sets. Issues in on Intuitionistic Fuzzy Sets and Generalized Nets, 11:1-8, 2014.

[12] K. Atanassov, E. Szmidt and J. Kacprzyk. On intuitionistic fuzzy pairs. Notes on Intuitionistic Fuzzy Sets, 19(3):1-13, 2013.

[13] K. Atanassov, V. Atanassova and G. Gluhchev. InterCriteria Analysis: ideas and problems. Notes on Intuitionistic Fuzzy Sets, 21(1):81-88, 2015.

[14] V. Atanassova, D. Mavrov, L. Doukovska and K. Atanassov. Discussion on the Threshold Values in the InterCriteria Decision Making Approach. Notes on Intuitionistic Fuzzy Sets, 20(2): 94-99, 2014.

[15] M. Birattari, T. Stutzle, L.Paquete, K. Varrentrapp, A racing algorithm for configuring metaheuristics, Proceedings of the Genetic and Evolutionary Computation Conference, pp. 11-18, 2002.

[16] Bonabeau E., Dorigo M. and Theraulaz G., Swarm Intelligence: From Natural to Artificial Systems, New York,Oxford University Press, 1999.

[17] M. Dorigo, L. Gambardella, Ant colony system : A cooperative learning approach to the traveling salesman problem, IEEE Transactions on Evolutionary Computation vol. 1, 53-66, 1996.

[18] Dorigo M, Stutzle T., Ant Colony Optimization, MIT Press, 2004.

[19] S. Fidanova, I. Lirkov, 3d protein structure prediction, Analele Universitatii de Vest Timisoara, vol. XLVII, pp. 33-46, 2009.

[20] S. Fidanova, An improvement of the grid-based hydrophobic-hydrophilic model, Int. J. Bioautomation, vol. 14, pp. 147-156, 2010.

[21] S. Fidanova, ACO algorithm with additional reinforcement, Int Conf. from Ant Colonies to Artificial Ants, Lecture Notes in Computer Science 2463, 292-293, 2003.

[22] S. Fidanova,K. Atanassov, P. Marinov, Generalized nets and ant colony optimization, Bulg. Academy of Sciences Pub. House, 2011.

[23] S. Fidanova, K. Atanassov, P. Marinov, Start strategies of ACO applied on subset problems, Numerical Methods and Applications, Lecture Notes in Computer Science, 6046, 248-255, 2011.

[24] S.Fidanova, K. Atanassov, P. Marinov, Intuitionistic fuzzy estimation of the ant colony optimization starting points, Large Scale Scientific Computing, Lecture Notes in Computer Science 7116, 219-226, 2012.

[25] S. Fidanova, O. Roeva and M. Paprzycki. InterCriteria Analysis of ACO Start Strategies, In Proceedings of the 2016 Federated Conference on Computer Science and Information Systems, Vol. 8, pages 547-550, 2016.

[26] Fidanova S. Hybrid Ant Colony Optimization Algorithm for Multiple Knapsack Problem. 5th IEEE International Conference on Recent Advances and Innovations in Engineering (ICRAIE), IEEE, 2021, DOI:10.1109/ICRAIE51050.2020.9358351, 1-5.

[27] Feuerman, Martin; Weiss, Harvey (April 1973). "A Mathematical Programming Model for Test Construction and Scoring". Management Science. 19 (8): 961-966

[28] D.E. Goldberg, B. Korb, K. Deb, Messy Genetic Algorithms: Motivation Analysis and First Results, Complex Systems, vol. 5(3), pp. 493 - 530, 1989. 
[29] D. Karaboga, B. Basturk, Artificial bee colony (ABC) optimization algorithm for solving constrained optimization problems, Advances in Soft Computing: Foundations of Fuzzy Logic and Soft Computing, LNCS 4529, 789-798, 2007.

[30] Kellerer H., Pferschy U., Pisinger D. (2004) Multiple Knapsack Problems. In: Knapsack Problems. Springer, Berlin,

[31] J. Kennedy, R. Eberhart, Particle Swarm Optimization. Proceedings of IEEE International Conference on Neural Networks. IV. pp. 1942-1948, 1995.

[32] S. Kirkpatrick, C.D. Gelatt, M.P. Vecchi, Optimization by simulated annealing Science (New York, N.Y.) (New York, N.Y.), 13 (220) (1983), pp. $671-680$

[33] G.Kochenberger, G.McCarl, F.Wymann, An heuristic for general integer programming, Decision Sciences vol. 5, 34-44, 1974.

[34] Jonas Krause, Jelson Cordeiro, Rafael Stubs Parpinelli, and Heitor Silverio Lopes. A survey ofswarm algorithms applied to discrete optimization problems. InSwarm Intelligence and Bio-Inspired Computation, pages 169-191. Elsevier, 2013

[35] E. L. Lawler, J. K. Lenstra, A. H. G. Rinnooy Kan, and D. B. Shmoys Sequencing and scheduling:algorithms and complexity. In S. C. Graves et al., editor,Handbooks in OR and MS, volume 4, pages445-522. Elsevier Science Publishers, 1993

[36] G.Leguizamon, Z.Michalevich, A new version of ant system for subse problems, Int. Conf. on Evolutionary Computations vol. 2, 1459-1464, 1999.

[37] Qing Liu, Tomohiro Odaka, Jousuke Kuroiwa, Haruhiko Shirai, and Hisakazu Ogura. A newartificial fish swarm algorithm for the multiple knapsack problem.IEICE TRANSACTIONS onInformation and Systems, 97(3):455-468, 2014

[38] S. Mirjalili, S. M. Mirjalili, A. Lewis, Grey Wolf Optimizer, Advances in Engineering Software, vol. 69, pp. 46-61, 2014.
[39] M.R. Mosavi, M. Khishe, G.R. Parvizi, M.J. Naseri, M. Ayat, Training multi-layer perceptron utilizing adaptive best-mass gravitational search algorithm to classify sonar dataset Archive of Acoustics, 44 (1) (2019), pp. $137-151$

[40] F. D. Murgolo. An efficient approximation scheme for variable-sized bin packing.SIAM Journal onComputing, 16(1):149-161, 1987.

[41] Schaffer, A. A., Yannakakis, M.: Simple Local Search Problems that are Hard to Solve. Society for Industrial Applied Mathematics Journal on Computing, Vol 20 (1991) 56-87.

[42] I.H. Osman, Metastrategy simulated annealing and tabue search algorithms for the vehicle routing problem, Annals of Operations Research, 41 (4) (1993), pp. 421-451

[43] S. Ravakhah, M. Khishe, M. Aghababaee, E. Hashemzadeh, Sonar false alarm rate suppression using classification methods based on interior search algorithm, International Journal of Computer Science and Network Security, 17 (7) (2017), pp. 58-65

[44] T. Stutzle, H. Hoos, Max min ant system, Future Generation Computer Systems, vol. 16, 889-914, 2000.

[45] P.A. Vikhar, Evolutionary algorithms: A critical review and its future prospects, Proceedings of the 2016 International Conference on Global Trends in Signal Processing, Information Computing and Communication (ICGTSPICC). Jalgaon, pp. 261-265, 2016.

[46] X.S. Yang, A New Metaheuristic Bat-Inspired Algorithm, Nature In spired Cooperative Strategies for Optimization, Studies in Computational Intelligence, 284: 65-74, 2010.

[47] X.S. Yang, Nature-Inspired Metaheuristic Algorithms, Luniver Press, 2008

[48] Andrew J Woodcock and John M Wilson. A hybrid tabue search/branch and bound approach tosolving the generalized assignment problem.European journal of operational research, 207(2):566-578, 2010 